\title{
Whole-body vibration training improves balance control and sit-to-stand performance among middle-aged and older adults: a pilot randomized controlled trial
}

\author{
Ming-Chen Ko ${ }^{1,2}$, Long-Shan Wü ${ }^{3}$ Sangwoo Lee ${ }^{2}$, Chien-Chun Wang ${ }^{1}$, Po-Fu Lee ${ }^{1,4}$, Ching-Yu Tseng ${ }^{1}$ \\ and Chien-Chang $\mathrm{Ho}^{1 *}$
}

\begin{abstract}
Background: Aging is associated with decreased balance, which increases falling risk. The objective of the current study was to determine the feasibility and effects of whole-body vibration (WBV) training on knee extensor muscle power, limits of stability, and sit-to-stand performance among community-dwelling middle-aged and older adults in the United States.

Methods: A randomized pilot study with participant blinding was conducted. Feasibility outcomes included recruitment and compliance rate. Twenty-nine community-dwelling older adults were randomly assigned to perform body-weight exercises with either an individualized vibration frequency and amplitude, a fixed vibration frequency and amplitude, or no vibration. Isokinetic knee extensor power, limits of stability, and sit-to-stand tests were conducted before beginning the exercises (baseline) and after 8 weeks of training.

Results: With a favorable recruitment rate (58\%) and compliance rates (attrition 9\%; adherence $85 \%$ ), the intervention was deemed feasible. The limits of stability endpoint excursion score for the individualized frequencyamplitude group was increased by $8.8(12.9 \% ; P=0.025)$ after training, and that group's maximum excursion score was increased by $9.2(11.5 \% ; P=0.006)$ after training. The average weight transfer time score was significantly decreased by $0.2 \mathrm{~s}$ in the fixed group. The participants in the individualized group demonstrated a significant increase (3.2\%) in weight rising index score after 8 weeks of WBV training.

Conclusions: WBV training is feasible for use with elderly people, and this study achieved good recruitment and compliance. The present paper suggests that 8 weeks of WBV training improves limits of stability and sit-to-stand performance. Future studies must determine whether WBV training improves other factors that affect posture control.
\end{abstract}

Trial registration: This study was registered at the Texas Woman's University Institutional Review Board TWU IRB 17632] on the 3rd of November 2014.

Keywords: Whole-body vibration training, Postural control, Balance, Limits of stability, Sit-to-stand test

\footnotetext{
* Correspondence: 093703@mail.fju.edu.tw

'Department of Physical Education, Fu Jen Catholic University, No. 510

Zhongzheng Road, Xinzhuang District, New Taipei City 24205, Taiwan

Full list of author information is available at the end of the article
} 


\section{Background}

One-third of community-dwelling individuals aged 65 years or older and approximately half of institutionalized people aged 80 years or older experience a fall each year [1]. Evidence also suggests that falling sideways from a standing position and landing on the trochanter region is strongly related to hip fracture [2]. Ten to $15 \%$ of falls result in injury or head trauma [3]; although only approximately $1 \%$ of falls cause hip fractures, more than $90 \%$ of hip fractures are due to a fall [4]. Therefore, falls should not be considered random events or accidents. Impairment of muscle strength and power of the lower extremities, balance/postural control, and of the walking ability are known to be substantial risk factors for falls, and these parameters have been found to become progressively more impaired with aging $[2,5]$. Therefore exercise should be generally accepted to effectively improve muscle strength, balance, and walking ability for the prevention of falls in older adults.

Whole-body vibration (WBV) training is a neuromuscular training modality that is used for strength training $[6,7]$. In recent years, a systematic review and metaanalysis study conducted by Rogan et al. [8] reported that WBV has been introduced as a training method to improve muscle power and strength in older adults. Tonic vibration reflex (TVR) is the most commonly accepted theory explaining the beneficial effects of WBV training on exercise performance and balance [9]. The mechanical stimuli generated by vibration platforms are transmitted to the body and stimulate muscle spindles. This activates alpha motor neurons, which then causes reflexive muscle contractions $[9,10]$. Increased muscle activity during WBV has been demonstrated using electromyography (EMG). For example, Hazell, Jakobi, and Kenno [11] reported that EMG activity was higher during the performance of dynamic semi-squats when WBV was involved than when it was not.

Delecluse et al. [6] compared the effects of WBV and resistance training on muscle strength, and found that 12 weeks of WBV (vertical sinusoidal vibration, 35$40 \mathrm{~Hz} ; 2.5-5 \mathrm{~mm}$ peak-to-peak amplitude) significantly increased isometric and dynamic knee extensor torque. The magnitude of the strength increment was comparable to resistance training at moderate intensity $(10-20$ repetitions per set). Furthermore, only the participants in the WBV group achieved a significant increase in countermovement jump height. In addition to its beneficial effects on muscle strength, WBV has also been observed to improve balance. Torvinen et al. [7] reported that a single bout of WBV (four sets, $60 \mathrm{~s}$ per set) significantly increased knee extensor strength, vertical jump height, and postural control in young individuals. However, these beneficial effects subsided $60 \mathrm{~min}$ after WBV.
Verschueren et al. [12] suggested that WBV training might also have positive effects on muscle strength and balance in postmenopausal women. After 6 months of WBV training, significantly increased isometric (16\%) and dynamic (10.6\%) knee extensor strength was discovered.

In another study, reduced anterior-posterior and mediolateral postural sway during arm abduction and anteflexion were used as indicators of improved postural control; the researchers determined that proprioceptive feedback from the ankle plays a crucial role in postural control [13]. Pollock et al. [14] reported that one session of WBV (five sets, $60 \mathrm{~s}$ per set) resulted in decreased cutaneous sensation. However, the results of balance tests did not reveal any significant changes. The explanation provided for this unchanged balance was that an insignificant tendency toward increased joint position sense might compensate for the decreased cutaneous sensation. Other studies have reported that after 812 weeks of WBV training, the knee movement detection threshold and joint position sense improved significantly in individuals with knee pathology $[15,16]$.

WBV training thus not only results in muscle function improvement, but also increases proprioceptor sensitivity. As previously discussed, resistance exercise training can improve muscle function and proprioceptive accuracy. However, elderly individuals were previously demonstrated to be at increased risk of falling or injury during strenuous load-bearing exercise [17]. Therefore, WBV training appears to be a promising alternative training modality. Unfortunately, the effects of this novel training modality on muscle function, balance, and proprioception, as determined from numerous studies, are inconsistent. This might be due to the different vibration frequencies, durations, and amplitudes used the studies. Furthermore, individual variability in the muscle damping coefficient might result in different people having distinct muscle responses even when following the same training protocol.

The objective of this pilot study was thus to use a randomized controlled trial to evaluate the feasibility and safety of using WBV training in the untrained elderly population and to determine the effects of body-weight exercise with an individualized WBV frequency on muscle function and balance. A fixed vibration frequency and no vibration were used as comparison treatments.

\section{Methods}

The design of this study followed that proposed by Thabne et al. [18], which described how to report the results of a pilot study and also stated that the main purpose of a pilot study is to determine the feasibility of a larger study. 


\section{Design}

A randomized controlled pilot study was conducted that enrolled elderly participants who were randomly assigned into the following three groups: the individualized frequency-amplitude group, fixed-frequency group, and control group. Participants were blinded regarding their group assignment and a familiarization session was held before any test or intervention. During the familiarization sessions, the participants' weight and height were obtained and the optimal vibration frequency and amplitude for each participant in the individualized frequency-amplitude group were determined. How the exercises should be performed on the vibration platform was also taught during the familiarization session. Baseline performance tests were conducted on the second visit of participants to the lab, and included limits of stability, sit-to-stand, and isokinetic knee extensor power tests. Post-intervention performance measurements (the same tests as baseline) were conducted 1 week after the 8 -week WBV intervention.

\section{Participants}

Participants were included if they fulfilled the following criteria: aged 55 years or above, able to stand with or without walking aids, and non-institutionalized. Individuals were excluded if they had a disease, were taking medications known to affect muscle strength, had had a recent fracture, had gall or kidney stones, had malignancies, were fitted with a cardiac pacemaker, or had already received WBV treatment.

\section{Randomization}

Randomization was achieved by assigning a blinded research assistant to draw pieces of paper inscribed with participants' names out of a box and divide the participants into the three groups (individualized frequency-amplitude, fixed-frequency, and control groups). The order of the measurements taken was also randomized for both the baseline and post-intervention tests. All participants gave written informed consent to the experimental procedure, which was approved by the Texas Woman's University Institutional Review Board (TWU IRB 17632) and was in accordance with the Declaration of Helsinki.

\section{Protocol}

The Power Plate pro5 (Power Plate North America Inc., U.S.A.) was used in this study. Its frequency can be adjusted from 25 to $50 \mathrm{~Hz}$ and its amplitude can be set at low or high ( 2 or $4 \mathrm{~mm}$ ). Five vibration frequencies $(30,35,40,45$, and $50 \mathrm{~Hz})$ and two amplitudes (low and high) were utilized to determine the optimal frequencyamplitude combination for each participant in the individualized frequency-amplitude group. Participants were instructed to stand on the platform with their knees bent to an angle of $45^{\circ}$. The participants could grasp the rails attached to the platform as needed.

One week before the participants started any intervention, the optimal frequency and amplitude for each participant in the individualized frequency-amplitude group was determined by monitoring the electromyogram of the vastus lateralis and medial gastrocnemius muscles. The Noraxon Telemyo 900 (Noraxon Inc., U.S.A.) was the EMG system used to analyze muscle activity in this study, and the Myoresearch XP software (Noraxon Inc., U.S.A.) was employed to analyze the signals collected by the EMG system. The surface electrodes (Noraxon Inc., U.S.A.) were disposable, selfadhesive $\mathrm{Ag}-\mathrm{AgCl}$ gel surface electrodes that had a diameter of $1 \mathrm{~cm}$ and an interelectrode distance of $2 \mathrm{~cm}$. The skin over the muscle groups of interest was sterilized with alcohol pads and excessive hairs were shaven off if needed before the attachment of the electrodes, which were placed on the surface of the muscle. The EMG signals were amplified $(1000 \times)$, band-pass filtered $(10-500 \mathrm{~Hz})$, rectified, smoothed (root-meansquare $=200 \mathrm{~ms}$ ), and sampled at $1000 \mathrm{~Hz}$ (MyoResearch XP Masters Edition 1.04, Noraxon Inc., U.S.A.) before any analysis was performed. The patella was chosen as the reference site because no muscle activity is detected at the patella.

The participants were required to undergo WBV for $20 \mathrm{~s}$ in an isometric half-squat position under the following conditions: vibration at $0,30,35,40,45$, and $50 \mathrm{~Hz}$, tested in a random order. Each trial was separated by 2 min of rest. Two amplitudes were also tested to determine the optimal combination for each participant; therefore, each participant in the individualized frequency-amplitude group was required to perform 10 WBV trials. The average EMG signal (measured in microvolts; $\mu \mathrm{V}$ ) during each $20 \mathrm{~s}$ trial was calculated, and the highest average EMG value obtained indicated the optimal intervention frequency and amplitude combination for each participant in the individualized frequency-amplitude group.

The participants in the two vibration groups underwent WBV three times a week for 8 weeks. There was a 24-48 h break between each vibration session (if the participant's vibration training was on Monday, Wednesday, and Friday, the participant was asked to take two days off and resume the next cycle on Monday. If the participant's vibration training schedule was on Tuesday, Thursday, and Saturday, the participant was asked to take two days off and resume the cycle on Tuesday). In each session, the participants performed five sets of WBV on the vibration platform, each set lasting $60 \mathrm{~s}$. To ensure multidirectional, balanced vibration loading of the lower 
extremities, the following exercises were performed on the vibration platform: a light squat (knee angle $45^{\circ}$ ), standing in the full upright position, standing on tiptoe (30 s) and then switching to the heels (30 s), alternating the body weight from one leg to another (30 s for each leg), and back to a light squat. The participants were allowed to take a 1-min break between sets, during which they were required to sit on a chair. The vibration frequency and amplitude were set individually for each participant in the individualized frequency-amplitude group, whereas the vibration frequency was set to the average vibration frequency used for the individualized group for the participants in the fixed-frequency group. Participants in the control group did not perform any training.

\section{Primary outcome: Criteria of success}

The criteria of success were based on the feasibility of the study protocol and focused on recruitment and compliance with the WBV training. The study was acceptable if one-third of the members of the LEAD-UP program at Texas Woman's University eligible for the training were recruited, there was a $15 \%$ attrition rate, and there was an $80 \%$ training attendance rate. These recommended values were based on a previous pilot study [19]. To calculate the attrition rate, the number of participants who did not complete the final followup measurements was determined. To calculate the training attendance rate, the total number of training sessions completed by each participant was recorded. Each participant could participate in a total of 24 WBV training sessions. For safety reasons, the participants were also interviewed before and immediately after each WBV training session regarding whether they had experienced feelings of stability, well-being, dizziness, or pain during the vibration.

\section{Secondary outcome}

The SMART Balance Master (Natus Medical Inc., U.S.A.) was used to measure the limits of stability (LOS) of the participants. The high validity and reliability values of measurement device and testing protocol have described and identified as being suitable for older adults [20]. This assessment determined their ability to voluntarily shift their center of gravity (COG) in eight directions in order to quantify the maximum distance they could travel without losing balance. The parameters that were measured were endpoint excursion (EPE), maximum excursion (MXE), and directional control (DCL). A sit-to-stand test was also performed on the SMART Balance Master. During the tests, the participants were required to be barefoot to eliminate the effects of different footwear. Foot alignment on the platform was determined according to each participant's height and followed the instruction provided in the SMART Balance Master manual. A human shape cursor was displayed on a computer screen, which represented the participants' COG. During the assessment, each participant was asked to move the cursor-using ankle strategies and weight-shifting only-to the highlighted targets. There were eight targets on the computer screen, positioned at $75 \%$ of the participants' estimated LOS. The participants were required to reach the highlighted targets as fast as possible; the maximal time allowed to reach the targets was $8 \mathrm{~s}$. Once the cursor reached the target, the participants were asked to hold that position until the trial was completed. The participants were told to get as close to the targets as they could without losing their balance or lifting their feet.

The following variables were used for the LOS test:

EPE: The distance traveled by shifting the COG to the highlighted targets; the endpoint was defined as the point at which the COG-shifting movement ceased.

MXE: The additional adjustments a participant makes to reach the targets.

DCL: The directional control represents whether the participants' movement was directly toward the targets; a score of $100 \%$ DCL indicates that no path deviation occurred.

The composite scores for EPE, MXE, and DCL for each of the eight directions were used for further data analysis.

Isokinetic power tests were performed on the dominant leg using a Biodex Multi-Joint System 3 dynamometer (Biodex Medical Systems, Inc., U.S.A). The measurement device and testing protocol have described and identified as being valid and reliable in older adults [21]. The knee extensor muscle power was tested at $60^{\circ} \mathrm{s}^{-1}$ and $180^{\circ} \mathrm{s}^{-1}$ and the rotational axis of the dynamometer was aligned with the lateral femoral condyle of the dominant leg. The knee extension began at a joint angle of $90^{\circ}$ and ended at $170^{\circ}$. The participants were required to perform one repetition of maximal knee extensor contraction at $60^{\circ} \mathrm{s}^{-1}$ and another repetition at $180^{\circ} \mathrm{s}^{-1}$. A $10-\mathrm{s}$ break was taken between repetitions. The whole procedure was repeated once, and the two trials were separated by 5 min of rest. The average power was used for statistical analysis.

The sit-to-stand (STS) test determines a participant's ability to transfer their COG from a seated to a standing position. The parameters measured were:

Weight transfer time (WTT): The time taken to transfer the COG forward, starting in a seated position and finishing with the complete body mass supported by the feet. 
Weight rising index (WTRI): The total amount of force generated during the rising phase.

Center of gravity sway velocity (COGSV): The percentage of body mass carried by each leg during the rising phase.

The participants were required to be barefoot to eliminate the effects of different footwear. To perform the STS test, the participants were directed to sit on a box (the height of the box was 12 in) with arms placed by their sides. The participants were then requested to stand up as quickly as possible without any help from their arms or any other physical aid. The test was performed three times and the average scores were used for data analysis.

\section{Statistical analysis}

All analyses were performed using IBM SPSS 19.0 (IBM Corp., Armonk, NY), and the significance level was set at $P<0.05$. Statistical analysis was performed using a two-way (vibration groups $\times$ time) multivariate analysis of variance. The dependent variables were (a) EPE, MXE, and DCL for the LOS test, and (b) WTT, WTRI, and COGSV for the STS test. Because some of the data were skewed rather than normally distributed, differences between groups and tests were then determined by use of the Wilcoxon signed rank test and Friedman test. The effect sizes (ESs) were calculated and expressed as $r$. For $r$, an ES of 0.1 is considered a "small" effect, around 0.3 a "medium" effect and 0.5 and above, a "large" effect [22].

\section{Results}

Figure 1 describes the flow of the participants through the study. All of them had been exercising at least two days per week for the preceding 6 months, and none were on any type of medication that would contaminate the data. The physiological characteristics of the participants are presented in Table 1.

\section{Recruitment, attrition, and adherence}

All of the participants were members of the LEADUP program at Texas Woman's University. The program had a total of 115 members, of which staff representatives estimated 60 were still active. A total of 35 individuals volunteered to participate in the study during the recruitment process, resulting in a recruitment rate of approximately $58 \%$. After they had completed a questionnaire during the screening interview, three participants were excluded because of the presence of an artificial cardiac pacemaker $(n=1)$, having a hip replacement that contained metal $(n=1)$, and having an inability to participate due to a scheduling conflict $(n=1)$. A total of 32 participants then signed the written informed consent, resulting in an inclusion rate-the proportion of participants invited to participate who enrolled-of approximately $91 \%$. However, three participants dropped out before or after the first WBV training session for the following reasons: the use of a portable oxygen concentrator and the participant's doctor suggesting no participation $(n=1)$; severe headache after the first WBV training session $(n=1)$; and cataract surgery $(n=1)$. A total of 29 participants completed all of the follow-up measurements (individualized frequency-amplitude group: $n=10$; fixed group: $n=9$; control group: $n=10$ ), resulting in an approximately 9\% attrition rate. The number of WBV sessions completed divided by the total number of training sessions offered indicated an excellent adherence rate of $85 \%$ to the study protocol over the 8 -week training period. No side effects related to the intervention were reported.

\section{Secondary outcomes Muscle power}

The baseline and post-WBV-training isokinetic power measurements of each group are presented in Table 2. Notably, there was no significant between-group difference in knee extensor muscle power at $60^{\circ} \mathrm{s}^{-1}$ and $180^{\circ} \mathrm{s}^{-1}$ at baseline or after 8 weeks of training.

\section{Effects of WBV training on LOS}

The baseline and posttraining results for EPE, MXE, and DCL are presented in Fig. 1. No baseline differences were observed in any of the LOS variables. EPE and MXE were significantly increased after 8 weeks of WBV training in the individualized frequency-amplitude group. The EPE for the individualized group at baseline was 68.0 , which had increased by $8.8(12.9 \% ; P=0.025)$ once the training had been completed. The MXE for the individualized group at baseline was 79.5, which had increased by $9.2(11.5 \% ; P=0.006)$ once the training had been completed. No significant changes in EPE or MXE were observed in the fixed-frequency and control groups. Additionally, the baseline and posttraining results for DCL did not reveal significant differences for any group.

\section{Effects of WBV on STS test}

The baseline and post-WBV-training STS results for each group are presented in Table 3. After 8 weeks of WBV training, the average WTT had decreased by $0.2 \mathrm{~s}$ in the fixed-frequency group, and there was a significant increase $(3.2 \%)$ in the WTRI among the participants in the individualized group. However, no significant change any group's COGSV was noted. 
Endpoint excursion (EPE)

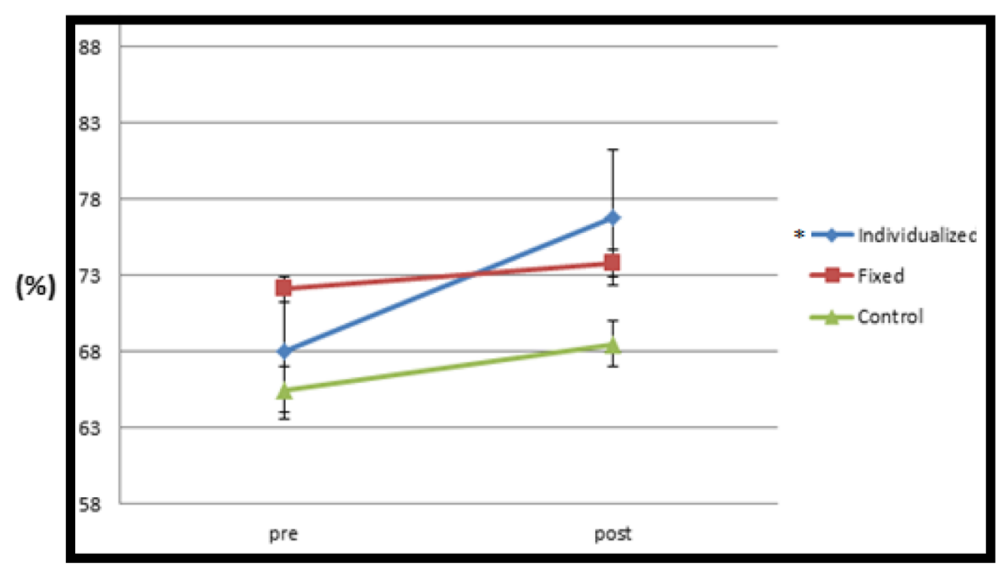

Maximum excursion (MXE)

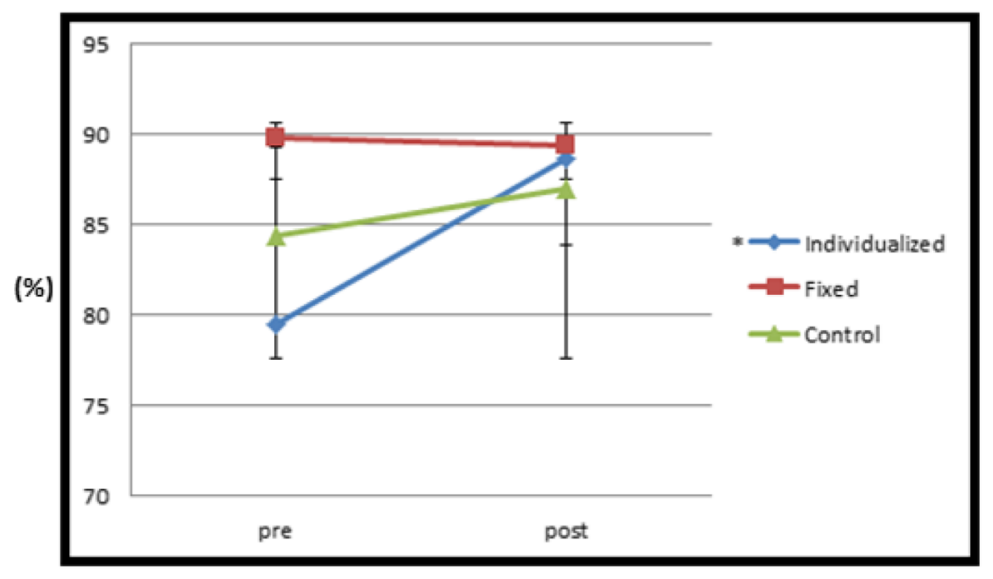

Directional control (DCL)

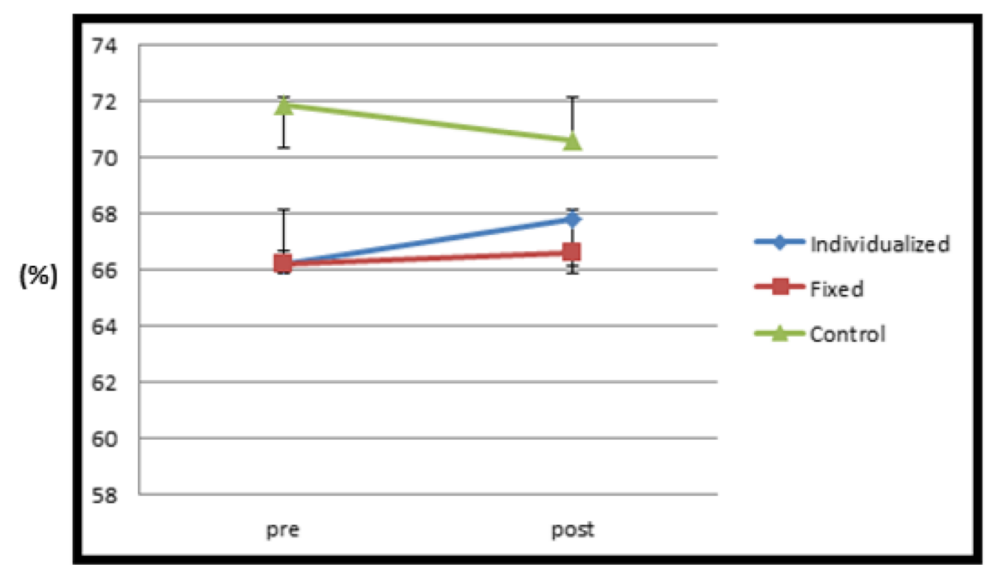

Fig. 1 The endpoint excursion (EPE), maximum excursion (MXE), and directional control (DCL) scores for pre and post whole-body vibration (WBV) training. *Post-test values are significantly higher than pre-test values at $P<0.05$ 
Table 1 Physiological characteristics of participants

\begin{tabular}{llll}
\hline & Ind $(n=10)$ & Fix $(n=9)$ & Con $(n=10)$ \\
\hline Age (years) & $69.2 \pm 7.0$ & $65.7 \pm 8.4$ & $66.0 \pm 4.1$ \\
Gender (Male/Female) & $3 / 7$ & $0 / 9$ & $7 / 3$ \\
Height $(\mathrm{cm})$ & $167.4 \pm 6.3$ & $161.2 \pm 2.3$ & $174.5 \pm 8.1$ \\
Weight $(\mathrm{kg})$ & $75.4 \pm 9.8$ & $65.1 \pm 12.1$ & $80.6 \pm 12.8$ \\
BMI $\left(\mathrm{kg} / \mathrm{m}^{2}\right)$ & $27.0 \pm 4.2$ & $25.0 \pm 4.7$ & $26.3 \pm 2.5$ \\
\hline
\end{tabular}

All values are expressed as means \pm SDs. Con control group, Fix fixed group, Ind individualized group, $S D$ standard deviation

\section{Discussion}

This randomized pilot study evaluated the feasibility of a WBV intervention in elderly people. In addition, the effect of 8 weeks of WBV training on muscle power, LOS, and STS performance was investigated.

The preliminary data provided useful information regarding the feasibility of WBV intervention for elderly people. Elderly individuals were successfully recruited, and no participant complained about and or reported side effects associated with the WBV training. This suggested that both individualized frequency-amplitude and fixed-frequency WBV training are feasible and safe intervention methods for the elderly population.

Improved postural control was also discovered in the current study. The data demonstrated that 8 weeks of WBV training using an individualized frequency and amplitude significantly improved participants' EPE, MXE, and WTRI. A significant decrease in WTT was also discovered in the fixed-frequency group. Notably, this improved LOS and STS performance was not accompanied by any significant changes in knee extensor power.

Reduced postural control and increased risk of falling due to age have been reported by previous studies $[23,24]$, and lower limb muscle strength is one of the factors correlated with postural control [25]. Resistance training has been reported to improve lower limb strength and also postural control in

Table 2 Isokinetic power measurement

\begin{tabular}{|c|c|c|c|c|c|}
\hline & Pre-test & Post-test & Change (\%) & $P$ & ES \\
\hline \multicolumn{6}{|c|}{60 deg./s (Watts) } \\
\hline Ind $(n=10)$ & $46.0 \pm 17.5$ & $50.1 \pm 20.2$ & $16.6 \pm 43.5$ & 0.252 & 0.588 \\
\hline $\operatorname{Fix}(n=9)$ & $45.8 \pm 27.1$ & $47.0 \pm 23.1$ & $13.6 \pm 33.0$ & 0.776 & 0.594 \\
\hline $\operatorname{Con}(n=10)$ & $66.0 \pm 22.3$ & $64.5 \pm 24.1$ & $-1.8 \pm 17.6$ & 0.606 & 0.600 \\
\hline \multicolumn{6}{|c|}{180 deg./s (Watts) } \\
\hline Ind $(n=10)$ & $63.8 \pm 26.6$ & $73.5 \pm 29.2$ & $18.9 \pm 27.2$ & 0.077 & 0.589 \\
\hline Fix $(n=9)$ & $67.6 \pm 40.1$ & $64.6 \pm 44.3$ & $-3.0 \pm 28.0$ & 0.670 & 0.594 \\
\hline $\operatorname{Con}(n=10)$ & $102.4 \pm 50.9$ & $93.6 \pm 50.2$ & $-9.3 \pm 11.2$ & 0.098 & 0.609 \\
\hline
\end{tabular}

All values are expressed as means \pm SDs. Con control group, ES effect size, Fix fixed group, Ind individualized group, SD standard deviation elderly people [26, 27]. Improved balance after WBV training in individuals with anterior cruciate ligament injury and older individuals has also been reported [28-30]. However, the aforementioned studies only used a fixed vibration frequency and amplitude in their training. Thus, the effects of individualized vibration frequency and amplitude on muscle strength and postural control still remain unclear.

LOS has been described as the greatest distance an individual can intentionally move when shifting their COG toward a given direction without losing balance, stepping, or grasping [31]. As the distance an individual can shift their COG decreases, their base of support-which is used to maintain dynamic balance during any type of standingbased physical activity-weakens [32]. Accordingly, an individual's LOS should be considered a critical prerequisite for the successful planning and execution of movements such as reaching forward to open a door or placing something on a shelf [33]. After 8 weeks of WBV training, only the participants in the individualized frequency-amplitude group demonstrated significant improvement in EPE and MXE. Bulat et al. [34] reported that 8 weeks of exercise training significantly improved EPE (from $40.9 \pm 9.2$ to $49.6 \pm 11.4$ ) and MXE (from $53.7 \pm 10.3$ to $63.9 \pm 13.3$ ). The training protocol adopted included lower extremity strengthening (stepping with an elastic band) and training for flexibility, coordination (dribbling soccer balls or completing an obstacle course), postural control (static standing with eyes closed), and gait (random, sudden changes in walking direction). The duration of each training period was $1 \mathrm{~h}$ (one session per week). The current study also identified improvement in EPE and MXE, obtained after 8 weeks of WBV training. The advantages of the WBV training protocol used in the present study are a shorter training duration per session $(10 \mathrm{~min})$ and a lower risk of falling during training sessions.

Several possible mechanisms may explain why individualized frequency-amplitude WBV training significantly improved LOS in the present study. For example, somatosensory stimulation (SSS) has been reported to trigger brain plasticity (modification of the maps in the brain cortex) [30, 35]. Van Nes et al. [30] indicated that WBV training (30 Hz, $3 \mathrm{~mm}$ peak-to-peak displacement, $45 \mathrm{~s}$ per set, four sets, $1 \mathrm{~min}$ pause between sets) significantly improved balance in individuals who had previously had a stroke. The authors suggested that WBV training was a strong SSS for both sides (paretic and unaffected) of the body that promoted brain plasticity and led to improved balance [30].

The improved LOS identified in the present study was supported by the study of Schuhfried et al. [29], which also used individualized WBV frequency. Those researchers began with a vibration frequency of $1 \mathrm{~Hz}$ and gradually increased it until each participant 
Table 3 Sit-to-stand measurements

\begin{tabular}{|c|c|c|c|c|c|}
\hline & Pre-test & Post-test & Change (\%) & $P$ & ES \\
\hline \multicolumn{6}{|l|}{ WTT (sec) } \\
\hline Ind $(n=10)$ & $0.4 \pm 0.2(0.3)$ & $0.4 \pm 0.2(0.3)$ & $-2.9 \pm 32.3$ & 0.536 & 0.540 \\
\hline Fix $(n=9)$ & $0.5 \pm 0.3(0.6)$ & $0.3 \pm 0.3(0.3)$ & $-36.0 \pm 29.9$ & $0.017^{*}$ & 0.674 \\
\hline $\operatorname{Con}(n=10)$ & $0.5 \pm 0.2(0.5)$ & $0.4 \pm 0.2(0.4)$ & $-7.4 \pm 48.8$ & 0.206 & 0.515 \\
\hline \multicolumn{6}{|c|}{ WTRI (\% of body weight) } \\
\hline Ind $(n=10)$ & $20.8 \pm 5.9$ & $24.0 \pm 6.0$ & $17.8 \pm 19.9$ & $0.025^{*}$ & 0.580 \\
\hline Fix $(n=9)$ & $22.4 \pm 9.1$ & $24.7 \pm 9.4$ & $13.8 \pm 31.9$ & 0.260 & 0.584 \\
\hline $\operatorname{Con}(n=10)$ & $24.7 \pm 9.3$ & $26.6 \pm 9.5$ & $11.0 \pm 22.0$ & 0.274 & 0.588 \\
\hline \multicolumn{6}{|l|}{ COGSV (deg/s) } \\
\hline Ind $(n=10)$ & $4.6 \pm 1.8$ & $4.7 \pm 0.8$ & $13.3 \pm 37.7$ & 0.868 & 0.573 \\
\hline Fix $(n=9)$ & $3.4 \pm 1.4$ & $4.2 \pm 1.1$ & $34.5 \pm 53.4$ & 0.054 & 0.566 \\
\hline $\operatorname{Con}(n=10)$ & $3.8 \pm 1.3$ & $4.5 \pm 0.9$ & $30.7 \pm 42.9$ & 0.072 & 0.534 \\
\hline
\end{tabular}

All values are expressed as means \pm SDs (medians). COGSV center of gravity sway velocity, Con control group, ES effect size, Fix fixed group, Ind individualized group, $S D$ standard deviation, WTRI rising index, WTT weight transfer time. *Post-test values are significantly different than pre-test values at $P<0.05$

(individuals with multiple sclerosis) could not tolerate a further increase; the maximum frequency reached was then used for treatment. Significant improvements in postural sensory organization and timed get-up-and-go were reported. The stimulation of pressure receptors (Merkel nerve endings, Meissner's corpuscles, Ruffini nerve endings), proprioceptors, and TVR are possible explanations for improved postural control [29, 36, 37]. However, caution is needed when interpreting the results of Schuhfried et al. [29] because the participants were only required to perform one bout of WBV exercise and the measurements were taken $15 \mathrm{~min}, 1$ week, and 2 weeks after the WBV exercise. Johansson [35] proposed that repeated stimulation of skin receptors promotes brain plasticity and that the cortical representation of the muscles involved will remain enlarged. Therefore, it is possible that the 8 weeks of WBV training used in the current study repeatedly stimulated the skin receptors and muscle spindle, which might explain why improved LOS was discovered.

Fixed-frequency and amplitude WBV training has also been reported to significantly improve balance [38]. For example, Cheung et al. [39] demonstrated that 3 months of WBV training $(20 \mathrm{~Hz}, 2 \mathrm{~mm}$ peak-to-peak displacement, 3 consecutive min on the vibration platform, three sessions per week) improved MXE (increased by $18.8 \% \pm 18.3 \%$ ) and DCL (increased by $4.3 \% \pm 19.6 \%$ ) in elderly women. Improved neuromuscular coordination may account for these improved MXE and DCL. In contrast with the study of Cheung et al. [39], the fixed-frequency and amplitude WBV training employed in the present study did not result in any meaningful changes in any of the balance variables. Identifying the exact mechanism that may account for why only participants in the individualized group exhibited balance improvements is not possible; however, a stronger SSS from the individualized vibration frequency and amplitude is a possible explanation because the vibration frequency and amplitude were individualized for each participant in the individualized group according to their EMG readings. Therefore, an individualized WBV protocol might enable the provision of a stronger stimulation to skin receptors, muscle spindles, and the vestibular system [29, 40].

Transferring the COG when moving from a sitting to a standing position is an essential daily movement and significant functional limitations can result if this ability is impaired [41]. STS tests have been used as a measurement for postural control, fall risk, lower limb strength, proprioception, and visual contrast sensitivity [42-44] for a variety of groups, such as individuals with arthritis, renal disease, stroke, and Parkinson disease, as well as older adults [45-48]. The transition from a sitting to a standing position changes the base of support from having three points to having two, which poses a challenge for dynamic stability and postural control [49]. A shorter STS time has been suggested to be an indication of higher postural and directional control [50]. Bhatt et al. [45] stated that the STS transition can be divided into two phases: movement preparation (the initiation of the movement; up until the lift-off) and execution (from the lift-off to the end of the movement). Individuals with Parkinson disease have lower hip flexion torques during the movement preparation phase and take longer to reach peak hip and knee extension torques during the execution phase, which leads to a longer completion time of the STS test [45].

Tung and Yang [51] reported that the significantly shorter STS time in individuals who had had a stroke after 4 weeks of STS training was due to higher hip extensor, knee extensor, and plantar flexor strength; similar results have also been observed after WBV 
training. Lee et al. [52] required participants (individuals with diabetic peripheral neuropathy) to perform WBV training $(15-30 \mathrm{~Hz}$, gradually increased from the first week to the sixth week; 2-mm peak-to-peak amplitude, three sets, 3 min per set) in addition to a balance exercise program (static and dynamic training). The results indicated that balance exercise training that included WBV resulted in significantly greater STS performance and postural control improvements compared with balance exercise training alone. Increased plantar flexor strength may have caused the improved performance. In the current study, 8 weeks of fixed-frequency WBV training significantly improved STS performance. Shorter WTT and higher WTRI after WBV training were indications of improved dynamic stability in the current study. Although one previous study [53] indicated that STS performance is related to lower limb muscle strength, concurrent improvement in knee extensor strength was not observed in the current study. Visual contrast sensitivity, lower limb proprioception, tactile sensitivity, and anxiety were also suggested as significant and independent predictors of STS performance [44]. Therefore, it is possible that the WBV protocol used in the current study improved factors related to STS performance other than muscle strength.

\section{Limitations}

There are some limitations of the present study to be discussed. First, this study attempted to determine the most effective WBV-training frequency and amplitude combination for each individual to improve their knee extensor muscle power and postural control. However, the specific frequency and amplitude identified for each participant in the individualized group were determined according to EMG readings obtained during light squats (at a knee angle of $45^{\circ}$ ). It is possible that the optimal vibration frequency and amplitude combination might vary according to different leg positions. In further studies, the frequency and amplitude combination for different leg positions should be determined. Second, the knee extensor was the only muscle group measured. Whether WBV training can improve the strength of plantar flexors and hip extensors was not addressed in the present study. More muscle groups related to postural control and STS performance should be measured. Third, the participants in the present study were members of the LEAD-UP program and were already performing exercise at least two times per week. Hence, the intensity of the WBV training may not have been high enough to induce any significant change in knee extensor strength. To isolate the effectiveness of WBV training on the measured variables, untrained elderly individuals should be recruited.

\section{Conclusions}

This pilot study demonstrated that the study protocol is feasible and safe for use with elderly individuals. Furthermore, the current study suggested that participants in the individualized frequency-amplitude group significantly improved their LOS and STS performance after 8 weeks of WBV training. Improved STS performance was also observed in the fixed-frequency group. However, 8 weeks of WBV training did not have any effect on knee extensor strength. Factors other than knee extensor strength-for example, improved hip extensor and plantar flexor strength, proprioception, and neuromuscular coordination-may account for the improved LOS and STS performance.

\section{Abbreviations \\ COG: Center of gravity; COGSV: Center of gravity sway velocity; DCL: Directional control; EMG: Electromyography; EPE: Endpoint excursion; ES: Effect size; LOS: Limits of stability; MANOVA: Multivariate analysis of variance; MXE: Maximum excursion; SD: Standard deviation; STS: Sit to stand; TVR: Tonic vibration reflex; WBV: Whole-body vibration; WTRI: Weight rising index; WTT: Weight transfer time}

\section{Acknowledgments \\ The authors would like to thank Beth Palmer the Pioneer Hall facility manager for providing the laboratory.}

\section{Fundings}

No external funding was sought or obtained for this study, which was undertaken for a Texas Woman's University Doctor in Sport Sciences dissertation.

Availability of data and material

All relevant material are presented in the present manuscript.

\section{Authors' contributions}

HCC participated in the design, conducted the statistical analyses, interpreted the data, and drafted the manuscript. KMC supervised the study, assisted in data interpretation, and critically reviewed the manuscript. WLS and LS helped in conducting the study and revising the manuscript. WCC, LPF and TCY helped to manage and analyze the data. All authors read and approved the final manuscript.

\section{Ethics approval and consent to participate}

The study was conducted in accordance with the Declaration of Helsinki, and all procedures have been approved by the Institutional Review Board of the Texas Woman's University (TWU IRB 17632). Written informed consent was obtained from each subject after a full explanation of the study.

Consent for publication

Not applicable.

\section{Competing interests}

The authors declare that they have no competing interest.

\section{Publisher's Note}

Springer Nature remains neutral with regard to jurisdictional claims in published maps and institutional affiliations.

\section{Author details}

${ }^{1}$ Department of Physical Education, Fu Jen Catholic University, No. 510 Zhongzheng Road, Xinzhuang District, New Taipei City 24205, Taiwan. ${ }^{2}$ Department of Kinesiology, Texas Woman's University, Denton, TX 762, USA. ${ }^{3}$ Graduate Institute of Sports Training, University of Taipei, Taipei City 11153, Taiwan. ${ }^{4}$ Graduate Institute of Sport Coaching Science, Chinese Culture University, Taipei City 11114, Taiwan. 


\section{Received: 3 December 2016 Accepted: 7 July 2017}

\section{Published online: 18 July 2017}

\section{References}

1. Tinetti ME, Speechley M, Ginter SF. Risk factors for falls among elderly persons living in the community. N Engl J Med. 1988;319(26):1701-7.

2. Runge $M$, Rehfled $G$, Resnicek $E$. Balance training and exercise in geriatric patients. J Musculoskelet Neuronal Interact. 2000;1(1):61-5.

3. Tinetti ME, Doucette J, Claus E, Marottoli R. Risk factors for serious injury during falls by older persons in the community. J Am Geriatr Soc. 1995; 43(11):1214-21.

4. Graafmans WC, Ooms ME, Hofstee HM, Bezemer PD, Bouter LM, Lips P. Falls in the elderly: a prospective study of risk factors and risk profiles. Am J Epidemiol. 1996;143(11):1129-36.

5. Asmussen E. Aging and exercise. Environ Physiol. 1980;3:419-28.

6. Delecluse C, Roelants M, Verschueen S. Strength increase after whole-body vibration compared with resistance training. Med Sci Sports Exerc. 2003; 35(6):1033-41

7. Torvinen $S$, Kannu P, Sievänen $H$, Järvinen TA, Pasanen $M$, Kontulainen S, Järvinen $T L$, Järvinen $M$, Oja $P$, Vuori I. Effect of a vibration exposure on muscular performance and body balance. Randomized cross-over study. Clin Physiol Funct Imaging. 2002;22(2):145-52.

8. Rogan S, de Bruin ED, Radlinger L, Joehr C, Wyss C, Stuck NJ, Bruelhart Y, de Bie RA, Hilfiker R. Effects of whole-body vibration on proxies of muscle strength in old adults: a systematic review and meta-analysis on the role of physical capacity level. Eur Rev Aging Phys Act. 2015;12:12. doi:10.1186/ s11556-015-0158-3.

9. Burke D, Hagbarth KE, Löfstedt L, Wallin BG. The responses of human muscle spindle endings to vibration during isometric contraction. J Physiol. 1976:261(3):695-711.

10. Hagbarth $\mathrm{KE}$, Eklund $\mathrm{G}$. Tonic vibration reflexes (TVR) in spasticity. Brain Res. 1966;2(2):201-3

11. Hazell T, Jakobi J, Kenno K. The effects of whole-body vibration on upperand lower-body EMG during static and dynamic contractions. Appl Physiol Nutr Metab. 2007;32(6):1156-63.

12. Verschueren SM, Roelants M, Delecluse C, Swinnen S, Vanderschueren D, Boonen S. Effect of 6-month whole body vibration training on hip density, muscle strength, and postural control in postmenopausal women: a randomized controlled pilot study. J Bone Miner Res. 2004;19(3):352-9.

13. Bloem BR, Allum JH, Carpenter MG, Honegger F. Is lower leg proprioception essential for triggering human automatic postural responses? Exp Brain Res. 2000;130(3):375-91.

14. Pollock RD, Woledge RC, Martin FC, Newham DJ. Effects of whole body vibration on motor unit recruitment and threshold. J Appl Physiol. 2012; 112(3):388-95. doi:10.1152/japplphysiol.01223.2010.

15. Moezy A, Olyaei G, Hadian M, Razi M, Faghihzadeh S. A comparative study of whole body vibration training and conventional training on knee proprioception and postural stability after anterior cruciate ligament reconstruction. Br J Sports Med. 2008;42(5):373-8. doi:10.1136/bjsm.2007. 038554.

16. Trans T, Aaboe J, Henriksen M, Christensen R, Bliddal H, Lund H. Effect of whole body vibration exercise on muscle strength and proprioception in females with knee osteoarthritis. Knee. 2009;16(4):256-61. doi:10.1016/j.knee. 2008.11.014

17. Kallinen M, Markku A. Aging, physical activity and sports injuries. An overview of common sports injuries in the elderly. Sports Med. 1995; 20(1):41-52.

18. Thabane L, Ma J, Chu R, Cheng J, Ismaila A, Rios LP, Robson R, Thabane M, Giangregorio L, Goldsmith CH. A tutorial on pilot studies: the what, why and how. BMC Med Res Methodol. 2010;10:1. doi:10.1186/1471-2288-10-1.

19. Rogan $S$, Radlinger $L$, Hilfiker $R$, Schmidtbleicher $D$, de Bie RA, de Bruin ED. Feasibility and effects of applying stochastic resonance whole-body vibration on untrained elderly: a randomized crossover pilot study. BMC Geriatr. 2015;15:25. doi:10.1186/s12877-015-0021-4

20. Liston RA, Brouwer BJ. Reliability and validity of measures obtained from stroke patients using the balance master. Arch Phys Med Rehabil. 1996;77(5):425-30.

21. Drouin JM, Valovich-mcLeod TC, Shultz SJ, Gansneder BM, Perrin DH. Reliability and validity of the Biodex system 3 pro isokinetic dynamometer velocity, torque and position measurements. Eur J Appl Physiol. 2004;91(1):22-9.
22. Corder GW, Foreman DI. Nonparameztric statistics for non-statisticans. A step-by-step approach. John Wiley \& Sons. Inc: Hoboken, New Jersey; 2009.

23. Chandler JM, Duncan PW, Kochersberger G, Studenski S. Is lower extremity strength gain associated with improvement in physical performance and disability in frail, community-dwelling elders? Arch Phys Med Rehabil. 1998;79(1):24-30

24. Rantanen T, Guralnik JM, Ferrucci L, Leveille S, Fried LP. Coimpairments: strength and balance as predictors of severe walking disability. J Gerontol A Biol Sci Med Sci. 1999;54(4):M172-6.

25. Brochu M, Savage P, Lee M, Dee J, Cress ME, Poehlman ET, Tischler M, Ades PA Effects of resistance training on physical function in older disabled women with coronary heart disease. J Appl Physiol. 2002;92(2):672-8.

26. Liu-Ambrose T, Khan KM, Eng JJ, Janssen PA, Lord SR, McKay HA. Resistance and agility training reduce fall risk in women aged 75 to 85 with low bone mass: a 6-month randomized, controlled trial. J Am Geriatr Soc. 2004;52(5):657-65

27. Hauer K, Specht N, Schuler M, Bärtsch P, Oster P. Intensive physical training in geriatric patients after severe falls and hip surgery. Age Ageing. 2002:31(1):49-57.

28. Roberts $D$, Andersoon G, Friden T. Knee joint proprioception in ACLdeficient knees is related to cartilage injury, laxity and age: a retrospective study of 54 patients. Acta Orthop Scand. 2004;75(1):78-83.

29. Schuhfried O, Mittermaier C, Jovanovic T, Pieber K, Paternostro-Sluga T. Effects of whole-body vibration in patients with multiple sclerosis: a pilot study. Clin Rehabil. 2005;19(8):834-42.

30. van Nes IJ, Geurts AC, Hendricks HT, Duysens J. Short-term effects of wholebody vibration on postural control in unilateral chronic stroke patients: preliminary evidence. Am J Phys Med Rehabil. 2004;83(11):867-73.

31. Li F. The effects of tai Ji Quan training on limits of stability in older adults. Clin Interv Aging. 2014;9:1261-8. doi:10.2147/CIA.S65823.

32. Islam MM, Nasu E, Rogers ME, Koizumi D, Rogers NL, Takeshima N. Effects of combined sensory and muscular training on balance in Japanese older adults. Prev Med. 2004;39(6):1148-55.

33. Melzer I, Benjuya N, Kaplanski J, Alexander N. Association between ankle muscle strength and limit of stability in older adults. Age Ageing. 2009; 38(1):119-23. doi:10.1093/ageing/afn249.

34. Bulat T, Hart-Hughes S, Ahmed S, Quigley P, Palacios P, Werner DC, Foulis P. Effect of a group-based exercise program on balance in elderly. Clin Interv Aging. 2007;2(4):655-60.

35. Johansson BB. Brain plasticity and stroke rehabilitation. The Willis lecture Stroke. 2000;31(1):223-30.

36. Griffin MJ. Handbook of human vibration. San Diego: Academic Press; 1996.

37. Ribot-Ciscar E, Roll JP, Gilhodes JC. Human motor unit activity during postvibratory and imitative voluntary muscle contractions. Brain Res. 1996;716(12):84-90.

38. Bogaerts A, Verschueren S, Delecluse C, Claessens AL, Boonen S. Effects of whole body vibration training on postural control in older individuals: a 1 year randomized controlled trial. Gait Posture. 2007;26(2):309-16.

39. Cheung WH, Mok HW, Qin L, Sze PC, Lee KM, Leung KS. High-frequency whole-body vibration improves balancing ability in elderly women. Arch Phys Med Rehabil. 2007;88(7):852-7.

40. Tommerdahl M, Delemos KA, Whitsel BL, Favorov OV, Metz CB. Response of anterior parietal cortex to cutaneous flutter versus vibration. J Neurophysiol. 1999;82(1):16-33.

41. Whitney SL, Wrisley DM, Marchetti GF, Gee MA, Redfern MS, Furman JM. Clinical measurement of sit-to-stand performance in people with balance disorders: validity of data for the five-times-sit-to-stand test. Phys Ther. 2005: 85(10):1034-45.

42. Csuka M, McCarty DJ. Simple method for measurement of lower extremity muscle strength. Am J Med. 1985;78(1):77-81.

43. Hesse S, Schauer M, Petersen M, Jahnke M. Sit-to-stand manoeuvre in hemiparetic patients before and after a 4-week rehabilitation programme. Scand J Rehabil Med. 1998;30(2):81-6.

44. Lord SR, Murray SM, Chapman K, Munro B, Tiedemann A. Sit-to-stand performance depends on sensation, speed, balance, and psychological status in addition to strength in older people. J Gerontol A Biol Sci Med Sci. 2002:57(8):M539-43.

45. Bhatt T, Yang F, Mak MK, Hui-Chan CW, Pai YC. Effect of externally cued training on dynamic stability control during the sit-to-stand task in people with Parkinson disease. Phys Ther. 2013:93(4):492-503. doi:10.2522/ptj.20100423. 
46. Bohannon RW. Alternatives for measuring knee extension strength of the elderly at home. Clin Rehabil. 1998;12(5):434-40.

47. Brunt D, Greenberg B, Wankadia S, Trimble MA, Shechtman O. The effect of foot placement on sit to stand in healthy young subjects and patients with hemiplegia. Arch Phys Med Rehabil. 2002;83(7):924-9.

48. Cheng PT, Liaw MY, Wong MK, Tang FT, Lee MY, Lin PS. The sit-to-stand movement in stroke patients and its correlation with falling. Arch Phys Med Rehabil. 1998;79(9):1043-6.

49. Mourey F, Pozzo T, Rouhier-Marcer I, Didier JP. A kinematic comparison between elderly and young subjects standing up from and sitting down in a chair. Age Ageing. 1998;27(2):137-46.

50. Lee CC, Wang RY, Yang YR. Correlations among balance and mobility measures for patients with stroke. FJPT. 2003;28(3):139-46.

51. Tung FL, Yang YR. Balance outcomes after additional sit-to-stand training in subjects with stroke: a randomized controlled trial. Clin Rehabil. 2010;24(6): 533-42. doi:10.1177/0269215509360751.

52. Lee K, Lee S, Song C. Whole-body vibration training improves balance, muscle strength and glycosylated hemoglobin in elderly patients with diabetic neuropathy. Tohoku J Exp Med. 2013;231(4):305-14.

53. Batista FS, Gomes GA, Neri AL, Guariento ME, Cintra FA, Sousa Mda L, D'Elboux MJ. Relationship between lower-limb muscle strength and frailty among elderly people. Sao Paulo Med J. 2012;130(2):102-8.

Submit your next manuscript to BioMed Central and we will help you at every step:

- We accept pre-submission inquiries

- Our selector tool helps you to find the most relevant journal

- We provide round the clock customer support

- Convenient online submission

- Thorough peer review

- Inclusion in PubMed and all major indexing services

- Maximum visibility for your research

Submit your manuscript at www.biomedcentral.com/submit
Biomed Central 